\title{
Opportunity to Grow and Explore: Lessons from a Bioinnovation Graduate Student Summer Internship Program at the U.S. Food and Drug Administra- tion
}

\author{
Dr. Anne-Marie Jacob Job, Tulane University
}

Anne-Marie Job received her doctorate in Biomedical Engineering from Tulane University in 2011. Soon thereafter, she assumed the role of Program Manager to a newly-minted Interdisciplinary Phd Program at Tulane in Bioinnovation. This graduate program fosters the design and development of innovative biomedical technologies and products through a balanced educational approach that combines strong foundations in science and engineering and direct collaboration with healthcare professionals and business and regulatory partners. Bioinnovation graduate students participate in transformative biotechnology development in collaborative teams that link partners from Tulane's Schools of Science \& Engineering, Medicine, Public Health \& Tropical Medicine, Business and Law. Additional business and regulatory training through local biotech incubators and accelerators as well as a summer internship at the US Food and Drug Administration help to sharpen student's entrepreneurial acumen and prepare them for advanced careers as leaders at the interface of academia and industry.

\section{Rebecca Zarch, SageFox Consulting Group}

Rebecca Zarch is an evaluator and a director of SageFox Consulting Group. She has spent the last 10 years evaluating projects supporting young adults moving through the STEM pipeline and workforce development projects. Rebecca's work often involves evaluations of innovative approaches to STEM education, typically at community colleges and graduate school programs. Rebecca received her MBA in non-profit management at the Heller School for Social Policy and Management and her M.Ed. from the Harvard Graduate School of Education.

\section{Dr. Alan R. Peterfreund, SageFox Consulting Group}

Alan Peterfreund is Executive Director of SageFox Consulting Group, an education research, evaluation located in Amherst Massachusetts. Alan has a Ph.D. in Geology from Arizona State University, and has been a research faculty member at Brown University. A career-shift in 1984 led to 16 years of consulting in the private and public sector with primarily emphasis on organizational change, quality management, and employee participation. Starting in 2000, Alan began to focus on supporting higher education partners in projects that address broadening participation in the sciences, graduate student development, curriculum innovation, instructional technology, teacher professional development and other education reforms. For the past five years, Alan has been the lead evaluator for Epicenter, an NSF-funded STEP Center focused on infusing entrepreneurship and innovation into undergraduate engineering education.

\section{Dr. Donald P. Gaver, Tulane University}

Donald Gaver is Chair of the Biomedical Engineering Department and Director of the Interdisciplinary Bioinnovation PhD Program at Tulane University. In addition, he directs research in Tulane's Biofluid Mechanics Laboratory, which aims to develop an understanding of the interrelationship between the mechanical and physicochemical behavior of biological systems. These investigations focus on the pulmonary system with the goal of developing improved therapies for pulmonary diseases such as infant and acute respiratory distress syndrome (RDS and ARDS) and the prevention of ventilator-induced lung injury (VILI). At present, his research focuses on study of the dynamic interplay between transport processes, interfacial phenomena and fluid stresses that alter the pulmonary epithelial structure and function during respiratory distress. In addition, his research extends to the design of optimized microfluidic devices for biosensor technology. These integrated studies bring together basic and applied scientists (including computational scientists), device developers and physicians to study problems of high clinical importance. 


\title{
Opportunity to grow and explore - Lessons from a Bioinnovation graduate student summer internship program at the U.S. Food and Drug Administration
}

\begin{abstract}
The development of biomedical technologies and devices and their translation to the healthcare market requires a strong foundation in science and engineering, an understanding of clinical need, and well-honed entrepreneurial skills. Tulane University's Bioinnovation PhD program was initiated in 2012 through the National Science Foundation's Integrative Graduate Education and Research Training (IGERT) grant mechanism to provide emerging entrepreneurial scientists and engineers with the skills needed to bring research from "bench to bedside." A hallmark of the program is a summer internship at the Food and Drug Administration (FDA) in the Division of Postmarket Surveillance - this is a Division in the Office of Surveillance and Biometrics of the Center for Devices and Radiological Health (CDRH). All degree candidates spend the summer working at the FDA in Silver Spring, MD after the first year of academic study to understand the practical and regulatory considerations that are required for the successful development of biomedical technologies and devices.
\end{abstract}

Over the past three years, twelve fellows in three cohorts have participated in this summer internship program. The internships were a first for FDA; as such, expectations about what could be accomplished over a 12-week period were minimal. What occurred in the first couple of years was remarkable: Bioinnovation fellows were given considerable latitude and opportunity to discover and work on real world issues that the FDA needed to address. Fellows matriculated through the FDA along a path of relatively unstructured discovery, wherein they learned about the agency and the processes and issues associated with product regulatory practices. Likewise, the agency came to realize the value of an exploratory approach to orientation and training that is currently under consideration for new FDA analysts.

The FDA internship has also proven to be influential to the professional growth of the graduate fellows. They found particular value in their role as "active witnesses" to the agency's Signal Review Meetings, where they were able to observe, reflect and follow up with professionals on the decision making process associated with the adverse effects of a medical device. These interactions taught them how to network and learn through observation and to work within the confines of a hierarchical non-academic organization. The process also challenged analysts to expand their understanding of what is possible by considering new approaches to confronting regulatory issues.

\section{Description of the Interdisciplinary PhD in Bioinnovation}

Tulane University's Interdisciplinary Bioinnovation PhD Program was initiated by a National Science Foundation Integrative Graduate Education and Research Training (IGERT) grant in 2012 that was awarded to faculty from the Schools of Science \& Engineering and Medicine in collaboration with the Schools of Public Health \& Tropical Medicine, Business, and Law. The Bioinnovation leadership created this graduate program to address the growing need for doctoral training of entrepreneurial scientists and engineers interested in developing biomedical technologies beyond academia. In addition, it was anticipated that this traineeship would foster collaborative interactions between faculty members at different schools and departments. 
Bioinnovation students (a.k.a., "fellows") come to Tulane from an array of traditional backgrounds ranging from biomedical engineering (most common) to chemistry, biology and computer science. They share a common interest in translational research, and while almost all have traditional research experience with two years or less of work experience, they are naïve about the complexities associated with bringing research from the bench to the bedside.

As such, the Bioinnovation Program requires that fellows not only become well-versed in human physiology and science and engineering fundamentals, they must also develop an understanding of the business, regulatory and administrative hurtles they will face in today's rapidly evolving healthcare industry. To this end, business and law classes have been integrated into the Bioinnovation curriculum to supplement a rigorous science and engineering course load, and fellows regularly participate in entrepreneurship-focused seminars, conferences and competitions. One additional critical component of their training is a 12-week summer internship at the US Food and Drug Administration (FDA) in Silver Spring, MD in the Division of Postmarket Surveillance, which is a Division in the Office of Surveillance and Biometrics of the Center for Devices and Radiological Health (CDRH). This internship provides fellows with a unique insider's view of the federal regulatory process. Herein we outline our experiences with the FDA internship and discuss its impact to-date on both the Bioinnovation fellows and our partners at the FDA.

\section{Structure of internship}

Since its inception in 2013, each summer has brought significant change to the FDA internship as the directors worked to create an experience that adds value both to Bioinnovation fellows and to the FDA. The internship evolved in direct response to evaluation feedback from the fellows and the FDA and has been tailored to the unique strengths of incoming student cohorts and the particular needs of the FDA at the time:

Year 1 (2013): This was a learning experience for everyone. The FDA worked to lay the groundwork for the internship program. The first cohort of fellows worked on IT infrastructure to build databases for particular devices. Although the fellows felt that these projects did not align well with their strengths, the work served to expose them to aspects of the FDA's regulatory process.

Year 2 (2014): Projects were designed to provide a deep-dive into a product area to expose the fellows to the challenges of big data. There was more room for collaboration than the previous year, with fellows applying similar techniques to manipulate and analyze their data while the motivation and outcome for each project was still unique. Student feedback suggested that the FDA still did not fully utilize their skill sets.

Year 3 (2015): The number of fellows participating doubled, which challenged the FDA to pull together a sufficient number of mentors who could provide engaging work to support the entire group. To address this issue, they designed open-ended projects for teams of fellows that focused on coding and longitudinal analyses. Mentors were contentexperts but not experts in the technical approach, and it was expected that they would 
work together with fellows to validate solutions. Fellows felt appropriately challenged and engaged with these projects.

In addition to the project-specific work, fellows tour FDA research labs through the Office of Science and Engineering Laboratories; attend 'Signal Meetings' during which FDA analysts discuss patterns of product error and make recommendations; and avail themselves to lectures and other professional development at the FDA.

Fellows have exceptional access to senior-level FDA employees in the Division of Postmarket Surveillance. They work directly with mentors whose positions range from branch directors to team leaders and analysts. In addition, they meet bi-weekly or weekly with the higher-ranked internship directors, Drs. Isaac Chang and Steven Chasin, Director and Deputy Director, respectively, of the Division of Postmarket Surveillance. At the end of the summer, fellows present their work to their mentors, directors and colleagues in the Division of Postmarket Surveillance.

It is worth noting that Bioinnovation students sacrifice a typically productive summer of research at Tulane to participate in the FDA internship. While their advisors have found that upon return these students' research productivity may be slightly behind their peers as a consequence of their internship, they return from the FDA with high motivation and are quick to reengage with their research. Advisors unanimously report on surveys that the internship program is highly valuable for the fellows' professional development.

\section{Evaluation Methods}

Each cohort of fellows was interviewed within four weeks of the FDA internship. Cohort 1 was interviewed as a group on-site. Fellows in cohorts 2 and 3 were interviewed individually by phone. The structured interview asked for a summary of the tasks assigned, feedback on their experience, and perceived value and challenges of the internship. Both the Director and Deputy Director of the Division of Postmarket Surveillance were also interviewed at the conclusion of each summer internship period, either in-person or by phone depending on the year and their availability.

In January of 2016, ten of the twelve fellows representing each cohort participated in a short phone interview to discuss lasting consequences of the internship on their academics, research and/or careers. The FDA Director of the Division of Postmarket Surveillance was interviewed to gauge changes in perspective on the value of the summer program to the agency.

\section{Value to Fellows}

The FDA internship is a huge draw to the Tulane Bioinnovation Program. Most of the fellows cite this component of Bioinnovation training as one of the key reasons they chose to attend Tulane. Immediately after the internship, fellows report that they most appreciated attending Signal Meetings (this has been a highlight for all three cohorts), their exposure to research carried out in the Office of Science and Engineering Laboratories, and their regular discussions with the Director of Postmarket Surveillance (and internship director) about the organizational hierarchy of the FDA and how it operates as a governmental agency. 
The experience at the FDA provides fellows with an in-depth understanding and appreciation of the regulatory process. Fellows consistently report that their familiarity with the regulatory process is of great value to their current research endeavors. Furthermore, they anticipate that this knowledge will serve them well in their future careers. They develop this insight primarily through interactions with their mentors and directors that teach them how to read and decipher reports and to navigate the FDA functional hierarchy - factors that strongly influence the FDA's final regulatory and recall decisions.

For many, the mentor-fellow relationship went far beyond their internship projects. Mentors helped to broaden the fellows' understanding of the FDA by introducing topics and considerations outside of the scope of their projects and connecting fellows with other employees both inside and outside of the Division of Postmarket Surveillance. Some fellows also made good use of their time with their mentors to discuss issues related to their own research and gain access to other individuals within the FDA who deal more directly with their area of interest. Not all fellows developed successful relationships with their mentors; rather, some were more reluctant to take their time and were sensitive to the fact that "mentoring cuts into their own work-time. They're analysts, so they're hardest worked people in the department."

Longer-term, fellows report that the effects of the FDA internship have been meaningful. The most commonly cited consequence has been a shift in how they approach their research project. In a program that has translational research as a goal, the added awareness of the regulatory process is considered to be tremendously beneficial. It helps inform the fellows' research design process from the outset for approval and scalability. One fellow said that when speaking with colleagues about their potential research topics, he now first questions "how will they get that through the FDA?" According to another student, "If I was doing a traditional [Biomedical Engineering] project I'd be ignorant of a lot of considerations when developing the project." Another noted that "learning how the FDA works has really guided the questions I need to ask going forward. Not so much with my research, but my business plan." Fellows also report that the internship has helped to "demystify" the typically intimidating regulatory process, since they now understand the procedures as well as the willingness of the FDA to work with companies to help them succeed.

Several fellows commented that they have learned the language of the FDA, making it easier for them to find answers to regulatory questions than it is for their colleagues. For several fellows this also has helped them participate in discussions with professionals and representatives from the FDA at conferences and seminars. Many attribute the internship with exposing the "black box" of the FDA. One student explained that because he could be a "fly on the wall" at a variety of meetings and was also able to delve deeply into the failure reports submitted to the FDA, he now has the advantage of understanding how the FDA reviews adverse events, makes final decisions, and manages everything from product labeling to product recalls.

Several fellows are poised to submit their own products to the FDA in the next year. They express confidence that their awareness of the approval process puts them in a unique position. For instance, they now know to look at the reports of similar products, which are publicly available, to glean what is/isn't being approved. They also have a better idea than most of which product area they should submit to for review, given that many of their products cross traditional 
boundaries. In addition, because there are typically only one or two people that deal with a given product area, professional relationships developed during their internship will likely prove useful.

As they work towards future careers, fellows anticipate that their time with the FDA will help to differentiate them from other candidates and thus will be a draw to future employers. A few have noted that while the internship helped them realize that they were less interested in a long-term FDA profession, they now understand that working with the FDA could be a valuable steppingstone in their career path. One fellow is already making use of the experience by providing consulting services to biotech start-ups who are in the process of developing their own regulatory plans.

\section{Value to FDA}

Evaluations of the FDA have indicated that the Bioinnovation fellows have introduced a different perspective that the internship director, Dr. Chang, stated "elevates our thinking in terms of possibilities and what kinds of approaches might work." Each year he has structured the fellow's summer projects to address particular challenges facing the agency. The outcome of students' work has lent insight into matters such as novel training practices and new approaches to managing and interpreting large volumes of data. Dr. Chang expressed, "we saw how students assembled information, and we looked to see if we could use some of the same approaches for on-going analysis."

During the first year of the internship, the agency was in the process of reflecting on its analyst training model. At the time, it took about four years to fully train analysts. For one, analysts must understand how to interpret incoming reports, which are often confusing and potentially misleading. In addition, they must understand the intricacies of the biotech industry and the often-convoluted evolution of the technologies they review. FDA administration realizes that extended analyst training is not a viable option, since personnel turnover rates are high. Thus, the fellows provided an opportunity to pilot small components of a new training model.

Bioinnovation fellows, particularly the third cohort, have provided the FDA with the opportunity to explore larger logistical questions that are beyond the FDA workforce's current capacity. Specifically, the student projects helped the FDA make progress on particularly vexing issues related to the use of computing and databases to manage their data. For example, it has long been a challenge to explain to manufacturers how to properly enter information into the FDA's reporting system. Furthermore, it is difficult to identify the specific software elements that developers should report. It is critically important to clarify and enhance the current database system to better accommodate the staggering volume and scope of incoming data. Because Bioinnovation fellows were able to lend an outsider's perspective while embedded within the FDA, the third cohort leveraged its programming strengths to build a system that bridged the manufacturer and software developer. Deputy Director Chasin reported that this group of fellows "developed tools that we can now refine and use."

The fellows, with few exceptions, were enthusiastic, curious and professional. According to Dr. Chang, "from the analyst perspective, having students ask questions forces you to rethink how you approach your own work. The fellows helped our analysts expand their thinking of what is 
possible. Being a co-developer allows them to be involved with the exploration. It opens up a new level of thinking for analysts."

\section{Challenges}

The internship program has been valuable to all stakeholders; nonetheless, significant issues have become apparent. For instance, some fellows tend to feel that the FDA has not properly gauged their technical and computing skills. They also typically come into the internship with limited work experience outside of the academic laboratory and can find it difficult to make the transition to the FDA's professional, hierarchal environment. The Bioinnovation Program and FDA recognize these issues and continue to work to improve the internship experience for all parties. Below are a number of descriptions of challenges that have arisen in this program.

\section{a. Setting expectations}

The experience for each cohort of fellows has varied based on the current pressing issues of the FDA. For example, while one cohort was privy to a major compliance issue, another had more access to the pre-market side of the organization, and the third saw a more analytic perspective. The maturity of the internship program itself also had an impact on the fellows' and FDA's experience. Specifically, with each new year the internship process has become more streamlined; the FDA has developed a better understanding of the fellows' potential; and more recent cohorts have entered the program with a fuller understanding of the organization. At the same time, fellows in later cohorts also tend to build expectations about their internship from previous years that can be misleading. For instance, access to research labs and other parts of the agency changed from one summer to the next based upon the relationships between divisions and their needs at the time and also on the persistence of a given group of students to forge meaningful connections outside of the Division of Postmarket Surveillance. Students do not always realize the multiple factors that play into shaping their experience or their capacity to, within reason, respectfully enhance their internship. This will be better communicated with future cohorts, and the FDA may initiate an orientation prior to beginning the FDA residency.

\section{b. Understanding fellow skill level}

The FDA has seen unprecedented growth in the number of reports submitted to the agency without increasing staff. Developing new techniques for working with big data in a different manner is a priority for the agency and a void that the FDA has limited resources to tackle. Bioinnovation fellows helped to address this need by prototyping methods to more efficiently manage this data onslaught. Many of the fellows, however, have noted a "generational" divide in computational savviness between themselves and the FDA employees. They believe that they have alternative experiences with analytic and computational tools than some FDA employees, either due to age and time in school or the nature of their work as engineers and researchers. This has left some interns with the feeling of being underutilized, and earlier cohorts in particular questioned the utility of their projects after their summer internship was complete. It is evident that communication between the FDA and interns is of paramount importance, while honoring the FDA hierarchical structure.

\section{c. Conflicts that arise from the non-academic environment}


Fellows come to Tulane with limited non-academic experience. For some, the FDA internship is their first time in a professional role, and the contrast between the academic and professional environments was a challenge. Also, Bioinnovation fellows tend to possess an entrepreneurial mindset that embraces the rapid translation of research to product; however this can cause conflict with the mission of a government agency such as the FDA. As one fellow noted in the follow up interview, "it wasn't an environment of 'hurry up and get stuff done' it was an environment of 'do things right."'

Dr. Chang noted that one fellow made the observation that 'in the workplace, organizations are becoming flatter.' However, Dr. Chang maintains that while this is true in the startup environment, it is not true in large government agencies such as the FDA. A novice intern may initially think solely about a problem and focus on only his/her needs towards completion of that task. With maturity they embrace the idea that they are at the FDA to also learn the important process by which the FDA completes its mission. It is this transition that provides the value proposition for the intern, because it teaches him/her to understand and empathize with the FDA's rational approach and the responsibility inherent in making critical and consistent decisions associated with healthcare.

At times, this struggle to assimilate into the agency workplace was pronounced. FDA representatives found that some fellows had not been adequately prepared with the professional skills expected by the FDA - including sensitivity to the hierarchy of the organization; proper dress and demeanor; and, perhaps most importantly, awareness of how this experience differs from the academic environment. At times, this may have held them back from making the most of this unique opportunity. In particular, Dr. Chang reports that the fellows seemed to pay "more attention to the substance than the process" thus missing the potential to understand the context of the FDA. They focused on technical solutions before they were fully aware of the purpose of their projects and how they fit into the bigger picture related to the FDA mission.

Similarly, fellows attended CDRH Branch Meetings, and some initially reported that they "weren't relevant" to their projects, missing the opportunity to network and learn from discussions. Dr. Chang elaborated that these students didn't think to explore such questions as: "How would you approach presenting to a group of people like this? How would you extract information from them? What would you learn from a leadership management perspective in terms of who is making/leading the conversations, how they're leading, how they get agreement, consensus, etc." However, once Drs. Chang and Chasin coached the fellows on this missed potential, "they found the meetings to be much more interesting and enjoyed observing people."

We note the dichotomy of perception is a specific value of the internship program. We believe that it provides an important rationale for bringing Bioinnovation fellows to the FDA, because it informs each participant (FDA and intern) of the important viewpoints they contribute to the collaboration. This serves as an important learning opportunity from which all participants can grow and provides a spectacular career development opportunity for the interns.

\section{Conclusions}


Upon reflection, Bioinnovation fellows mutually attest to the value of the internship for increasing their awareness of and patience for bureaucracy and their general understanding of the federal regulatory and oversight process. The internship experience has provided an exceptional opportunity that leads fellows to appreciate the tremendous efforts of the agency and what it takes to bring about change in such a large, complex organization. Finally, and most critical to their current training and future careers, the fellows believe that their experiences at the FDA have better equipped them to fast-track the translation of their research from the academic laboratory to the medical arena. While both fellows and FDA employees have faced challenges in developing and improving this internship program, all participants strongly believe that the program has been of exceptional value for career development and advancement of the FDA.

\section{Acknowledgements}

Rebecca Zarch and Anne-Marie Job share responsibilities as lead authors of this manuscript.

The authors gratefully acknowledge our collaborators at the Food and Drug Administration. In particular, we are indebted to Drs. Isaac Chang and Steve Chasin for working with the Bioinnovation Program to establish, organize and manage this summer internship program. Drs. Chang and Chasin are Director and Deputy Director, respectively, of the Division of Postmarket Surveillance in the Office of Surveillance and Biometrics of the FDA's Center for Devices and Radiological Health.

The Bioinnovation Program was established with the support of a National Science Foundation Integrative Graduate Education and Research Training grant, DGE-1144646. 